\title{
LOS RESTOS ÓSEOS HUMANOS DE LA COLECCIÓN DE LA MISIÓN SALESIANA "LA CANDELARIA" (RÍO GRANDE, ARGENTINA)
}

\author{
JORGE A. SUBY* Y RICARDO A. GUICHÓN",*
}

\begin{abstract}
RESUMEN
La colección de restos óseos humanos del Museo de la antigua Misión Salesiana de Río Grande, como otras colecciones de Patagonia Austral, es en parte el resultado de donaciones y recuperaciones realizadas por aficionados y personal del museo en diferentes épocas. Estos materiales generan información sobre la variedad de situaciones que se presentan en la región, independientemente de que sus conjuntos no puedan ser asociados en la mayoría de los casos a un sitio en particular. Dentro de este marco, el objetivo del presente informe es comunicar los resultados obtenidos a partir del estudio de la colección de restos óseos humanos depositados en la antigua misión salesiana "La Candelaria" (Río Grande, Tierra del Fuego, Argentina). Estos análisis consistieron en la cuantificación e identificación de los elementos presentes en cada una de las cajas que componen la colección, la determinación del número mínimo de individuos, la composición sexo-etaria de la muestra y la descripción e interpretación de lesiones patológicas. En los casos posibles, sobre la base de información documental asociada, se determinó el lugar de procedencia de los restos óseos. Asimismo, para uno de los individuos se obtuvo un fechado radiocarbónico y valores de isótopos estables. La colección está compuesta por 14 cajas con restos óseos humanos, con un total de 1088 elementos y un número mínimo de 51 individuos (43 adultos y 8 subadultos). Desde un punto de vista paleopatológico, se identificó la predominancia de lesiones degenerativas articulares y algunos casos de interés para la región por su escasa documentación, particularmente los asociados a hechos de violencia interpersonal. Las tareas de conservación de colecciones, que requieren además constantes monitoreos y mantenimiento, representan un aspecto no siempre tenido en cuenta por los proyectos de investigación y que condiciona la información potencialmente disponible. Aunque frecuentemente las soluciones a los problemas de conservación no son simples, dado que en general se carecen de recursos adecuados, son tareas imprescindibles para posibilitar el mantenimiento de las colecciones de restos óseos humanos como patrimonio no renovable.
\end{abstract}

PALABRAS CLAVES: restos óseos humanos, Misión Salesiana de Río Grande, conservación, paleopatología. 


\section{ABSTRACT}

The human bone remains collection of the Museum of the Salesian Mission of Rio Grande, as other Patagonian collections, is the result of donations and recoveries of skeletons made at different times by amateurs and personnel of the museum. This collection provides information about the variety of situations of human bone record in the region, despite the fact that no contextual references are available. Therefore, it is important to have information about its composition which could be contrasted with remains obtained from systematic excavations. In this context, the objective of this paper is to communicate the results from the study of the human bone remains collection of the antique Salesian mission "La Candelaria" (Rio Grande, Tierra del Fuego, Argentina). These results include some preventive conservation tasks, the identification of the elements, determination of minimum number of individuals, sex-age composition and identification of pathological lesions. In some cases it was possible to obtain information of the remains' site of origin. The collection is composed by 14 boxes with 1088 human elements and 51 individuals, 43 of them are adults and 8 subadults. From a paleopathological point of view, the studies showed a predominance of degenerative joint diseases and revealed some cases of violence and social tension, scarcely reported in this region. The conservation of collections is an aspect which is not always included as part of research projects and that implies a potential source of information. Although the resolution of conservation problems is usually complex, the conservation of human bone collections is a central key to maintain this kind of record throughout time.

KEYWORDS: human bone remains, Salesian Mission of Rio Grande, conservation, paleopathology.

\section{INTRODUCCIÓN Y METODOLOGÍA}

Los estudios de las colecciones de restos humanos que no surgen de excavaciones sistemáticas han sido la base para los estudios de distancia biológica y otras investigaciones de Antropología Biológica en Patagonia. El grado de resolución a que arriban estos estudios (independientemente del problema tratado) está condicionado por la calidad de las muestras en cuanto a su ubicación contextual. Sin embargo, se trata de colecciones que en muchos casos carecen de esta información y sobre las cuales se aplican métodos de relevamiento y diseños estadísticos altamente complejos. En el caso de los estudios de la salud en el pasado, qué es posible preguntarle a una colección de estas características constituye un desafío complejo y variado, dependiente en gran medida de los objetivos que cada proyecto esté formulando. Entendemos que el caso específico de los estudios paleopatológicos supone una vinculación constante y recíproca entre el investigador y sus interpretaciones. En nuestro caso particular planteamos las colecciones como generadoras de expectativas generales y los restos recuperados sistemáticamente en el contexto de proyectos de investigación como situaciones de mayor control a partir de las cuales ajustar y mejorar esas expectativas.

La colección del Museo de la antigua Misión Salesiana de Río Grande, como otras colecciones de Patagonia Austral, es el resultado de donaciones y recuperaciones realizadas por aficionados y personal del museo en diferentes épocas (padre Ticó com. per.). En la década del 80 uno de nosotros (RAG) visitó el Museo y realizó un relevamiento métrico de los cráneos (Cocilovo y Guichón 1985/86) cuando aún se encontraban expuestos en vitrinas del museo (tanto en el edificio central como en el edificio de la capilla). En la década del 90 nuevamente visitamos la colección y realizamos un relevamiento de indicadores de estilo de vida de algunos restos (Guichón 1994). Algunos de los elementos que componen esta muestra fueron incorporados en estudios más extensos por diferentes especialistas que visitaron la isla (García Moro et al. 1997). Paralelamente al trabajo de relevamiento, en cada una de nuestras visitas se fueron realizando inventarios y algunas tareas de ordenamiento y conservación preventiva.

Hace dos años iniciamos el estudio del cementerio de la antigua Misión Salesiana de Río Grande en el marco de un proyecto orientado a estudiar la 
salud en momentos de contacto interétnico. Aquí la información documental y la excavación del cementerio aparecen como el objetivo central de nuestro trabajo. Sin embargo el estudio de las colecciones disponibles para la región y en este caso en particular la que se encuentra depositada en los reservorios del Museo Salesiano de Río Grande, generan información que nos orientan sobre la variedad de situaciones que se presentan en la región. Se trata de una muestra con información "promediada" de individuos que que fueron recuperados, en su mayoría, en un espacio próximo a la misión el cual es difícil de acotar. A esto debemos agregar otras cuestiones interesantes que ayudaron a reconstruir la ubicación y algunos aspectos de las condiciones de hallazgos en sectores para los cuales no contamos con información.

Dentro de este marco, el objetivo del presente informe es comunicar los resultados obtenidos a partir del estudio de la colección de restos óseos humanos depositados en la antigua Misión Salesiana "La Candelaria" (Río Grande, Tierra del Fuego, Argentina). Los trabajos formaron parte de las tareas incluidas en el proyecto de investigación "Paleoepidemiología del Contacto Aborigen Europeo en Patagonia Austral" (PICT 01520). Los análisis consistieron en la cuantificación e identificación de los elementos presentes en cada una de las cajas que componen la colección. En los casos posibles se obtuvo además información del lugar de procedencia de los restos óseos a través de entrevistas con el Padre Ticó y el seguimiento y análisis de información asociada a algunos de los restos. Para uno de los individuos se obtuvo un fechado radiocarbónico y valores de isótopos estables a partir de análisis realizados sobre una costilla, seleccionada por su buen estado de conservación y ausencia de lesiones patológicas.

La estimación del número probable de individuos que componen la colección se realizó mediante el método denominado Most Likely Number of Individuals (MNLI), propuesto por Adams y Konigsberg (2004). Este método de cuantificación para restos mezclados, al igual que el Lincoln Index (LI), mejora el potencial estadístico de estimación del método Minimum Number of Individual (MNI) empleado frecuentemente, debido a que se basa tanto en el número de elementos apareados como desapareados, es decir la comparación de elementos derechos e izquierdos para decidir si pertenecen a un mismo individuo (Adams y Konigsberg 2004). En este caso, para la estimación del NMLI se empleó una automatización disponible libremente en http:// konig.la.utk.edu/MLNI.html, publicada por uno de los autores originales. La cuantificación del MLNI se realizó para cada caja en forma individual, discriminándose entre adultos y subadultos. Asimismo, se efectuó también la estimación mediante el método de MNI, contabilizándose el elemento más frecuente presente en cada una de las cajas que componen la colección, y posteriormente se comparó con los resultados obtenidos con el método MLNI.

La colección está compuesta por cajas en las cuales se encuentran restos correspondientes a un sólo individuo y cajas con elementos mezclados pertenecientes a varios esqueletos. Para los restos que pudieron ser discriminados como parte de un mismo individuo se estimó el sexo según la morfología craneana (proceso mastoides, cresta nucal, glabela, margen supraorbitario y robustez de la mandíbula) y pélvica (características de la rama isquiopubiana, concavidad subpúbica y escotadura ciática mayor) propuestas en Buikstra y Ubelaker (1994). Para la estimación de la edad de los adultos se siguieron los métodos de Todd y Suchey-Brooks (en Buikstra y Ubelaker 1994) para la metamorfosis de la sínfisis púbica; el método de Iscan et al. (1984) para la metamorfosis del extremo esternal de la cuarta costilla y el método de Lovejoy et al. (1985) para la superficie articular de la pelvis. Cada método fue aplicado sólo en los casos en los cuales los rasgos diagnósticos se encontraron presentes y en buen estado de conservación. Para el caso de los subadultos y adultos jóvenes, la edad se estimó siguiendo los métodos de Ubelaker (1989), para la erupción dental y el patrón de cierres epifisarios (Buikstra y Ubelaker 1994). La edad final para cada caso se estimó como la más probable de acuerdo a los métodos empleados. Por otra parte, en las cajas donde se encuentran elementos mezclados correspondientes a más de un individuo, se estimó el sexo para cada elemento según los métodos propuestos por Bass (1985). Para la estimación de la edad, se aplicaron los métodos descriptos más arriba para cráneos, mandíbulas, pelvis y huesos largos. En los casos en que ningún elemento diagnóstico permitió esta clasificación, simplemente se identificaron como adultos o subadultos. Tal como comentan Buikstra y Ubelaker (1994:9) En conjuntos mezclados, el 
observador frecuentemente sólo puede distinguir restos maduros de inmaduros; rara vez pueden ser identificados infantes, niños, adolescentes $y$ adultos de varias edades (la traducción es nuestras).

Finalmente, cada elemento fue inspeccionado desde un punto de vista paleopatológico, considerando los conocimientos de anatomía ósea normal según Gray (1949). Las lesiones patológicas identificadas fueron registradas siguiendo las metodologías descriptivas y de diagnóstico diferencial propuestas por Campillo (2001), sugiriendo probables causas etiológicas. En algunos elementos se realizaron estudios radiográficos en el Hospital de Río Grande.

\section{RESULTADOS}

La colección que motiva este trabajo está compuesta por 14 cajas con restos óseos humanos. $\mathrm{Al}$ momento del inicio de los trabajos los restos se encontraban en cajas con los elementos envueltos en papel y apilados de manera que implicaban riesgos para su conservación. Como una mejora en esta situación, los elementos de cada caja fueron reordenados, protegidos con bolsas y papel libres de ácido e inventariados (Figura 1). En total fueron contabilizados un total de 1088 especímenes óseos, con un alto número de especimenes por cada caja (Tabla 1). Sólo algunas cajas presentaron un bajo número de especímenes (Cajas Nro. 9, 10 y 11), las cuales contienen únicamente cráneos. En general, los restos óseos poseen un buen estado de conservación, salvo algunos casos en los que los elementos más frágiles (e.g. costillas) se encuentran altamente fragmentados. En relación con la información contextual
Tabla 1. Número de restos óseos humanos presentes en las cajas que componen la colección depositada en la Misión Salesiana de Río Grande.

\begin{tabular}{|c|c|}
\hline Numero de Caja & Cantidad de Huesos Humanos \\
\hline Caja 1 & 101 \\
\hline Caja 2 & 169 \\
\hline Caja 3 & 172 \\
\hline Caja 4 & 91 \\
\hline Caja 5 & 92 \\
\hline Caja 6 & 62 \\
\hline Caja 7 & 103 \\
\hline Caja 8 & 26 \\
\hline Caja 9 & 5 \\
\hline Caja 10 & 6 \\
\hline Caja 11 & 6 \\
\hline Caja 12 & $\mathbf{7 9}$ \\
\hline Caja 13 & 46 \\
\hline Caja 14 & 130 \\
\hline TOTAL & $\mathbf{1 0 8 8}$ \\
\hline
\end{tabular}

disponible, sólo los restos contenidos en cuatro cajas pudieron ser asociados a un lugar aproximado de recuperación. Los restos de las cajas Nro. 2 y Nro. 5 provienen de las proximidades de la meseta de la Laguna Pali Aike (Santa Cruz) según anotaciones vinculadas a los restos. Los restos de la Caja Nro. 3 provienen de la Estancia Sofía (Tierra del Fuego) mientras los restos de la caja Nro. 14 provienen de la estancia San Julio (Tierra del Fuego). Este último representa un caso particularmente relevante por tratarse de uno de los pocos restos óseos humanos hallados en el sector interior del norte de la isla grande de Tierra del Fuego.

Respecto de la composición de la colección, 10 cajas (71\%) contienen restos pertenecientes a más
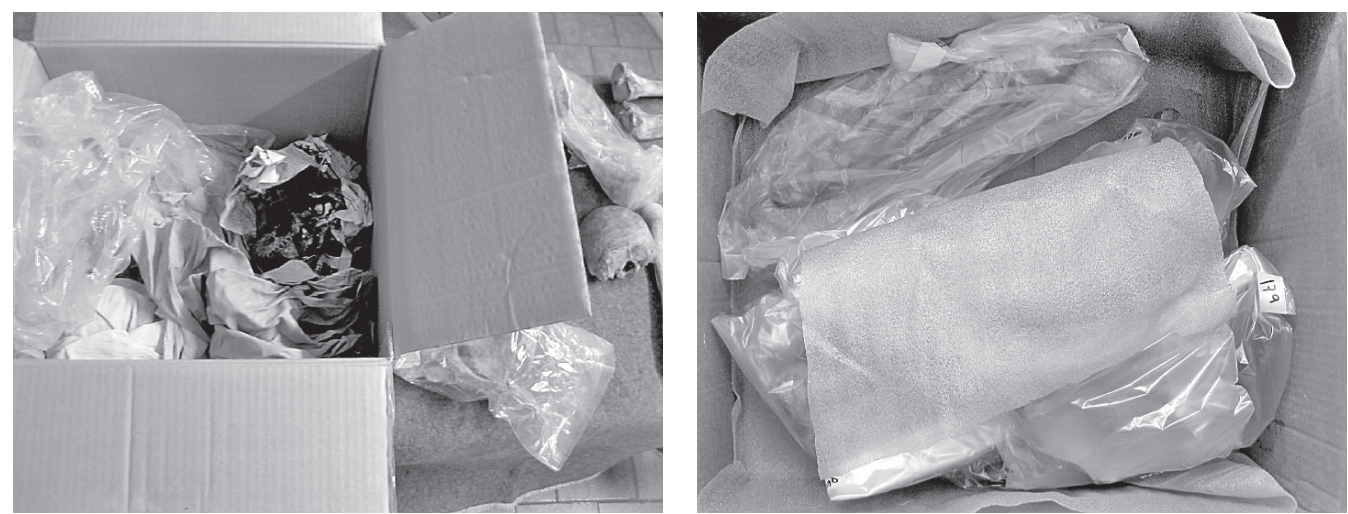

Fig. 1. A la izquierda, situación original de depósito de los elementos que componen la colección de la antigua Misión Salesiana de Rio Grande. A la derecha, elementos protegidos con papel y bolsas libres de acido. 
Tabla 2. Composición de los restos óseos humanos contenidos en cada una de las cajas de la colección de la Misión Salesiana "La Candelaria". Se presentan las estimaciones de MLNI y MNI así como los elementos a partir de los cuales fueron calculados en las cajas con más de un individuo, así como las edades y sexos probables estimados para cada caso.

\begin{tabular}{|c|c|c|c|c|c|}
\hline \multirow{2}{*}{ Caja Nro. } & \multirow{2}{*}{ MLNI / NMI } & \multirow{2}{*}{ Elemento/Lateralidad } & \multicolumn{2}{|c|}{ Composición } & \multirow{2}{*}{ Procedencia } \\
\hline & & & Sexo & Edad & \\
\hline \multirow{6}{*}{1} & \multirow{6}{*}{$6 / 6$} & \multirow{6}{*}{ Cráneos } & $\mathrm{M}$ & $20-35$ & \multirow{6}{*}{ Desconocida } \\
\hline & & & $M$ & $20-35$ & \\
\hline & & & $\mathrm{F}$ & $20-35$ & \\
\hline & & & $\mathrm{F}$ & $20-35$ & \\
\hline & & & I & $20-35$ & \\
\hline & & & I & $20-35$ & \\
\hline \multirow{6}{*}{2} & \multirow{6}{*}{$6 / 6$} & \multirow{6}{*}{ Humeros/derechos } & $\mathrm{M}$ & $35-50$ & \multirow{6}{*}{ Pali Aike } \\
\hline & & & $\mathrm{M}$ & $20-35$ & \\
\hline & & & $\mathrm{F}$ & $35-50$ & \\
\hline & & & I & $15-18$ & \\
\hline & & & I & $15-18$ & \\
\hline & & & I & $10-12$ & \\
\hline \multirow{3}{*}{3} & \multirow{3}{*}{$3 / 3$} & \multirow{3}{*}{ Clavículas/derechas } & I & $35-50$ & \multirow{3}{*}{ Desconocida } \\
\hline & & & I & $35-50$ & \\
\hline & & & I & Subadulto & \\
\hline \multirow{4}{*}{4} & \multirow{4}{*}{$4 / 4$} & \multirow{4}{*}{ Clavículas/derechas } & $\mathrm{M}$ & $35-50$ & \\
\hline & & & $\mathrm{M}$ & $35-50$ & \\
\hline & & & $\mathrm{M}$ & $35-50$ & Ea. Sofia \\
\hline & & & $\mathrm{M}$ & subadulto & \\
\hline & & & $\mathrm{M}$ & $35-50$ & \\
\hline & & & $\mathrm{M}$ & $35-50$ & \\
\hline 5 & $4 / 4$ & Húmeros/izquierdos & $\mathrm{F}$ & $35-50$ & Pali Aike \\
\hline & & & I & 14-19 & \\
\hline & & & $\mathrm{M}$ & $35-50$ & \\
\hline & & & $\mathrm{M}$ & $35-50$ & \\
\hline 6 & $5 / 5$ & Húmeros/derechos & $\mathrm{F}$ & $20-35$ & Desconocida \\
\hline & & & I & Adulto & \\
\hline & & & I & Subadulto & \\
\hline & & & $\mathrm{M}$ & $35-50$ & \\
\hline & & & $M$ & $35-50$ & \\
\hline & & & $\mathrm{M}$ & $35-50$ & \\
\hline 7 & $7 / 7$ & Tibias/ izquierdas & $\mathrm{M}$ & $20-35$ & Desconocida \\
\hline & & & $\mathrm{I}$ & Adulto & \\
\hline & & & I & $6-8$ & \\
\hline & & & I & $0-3$ & \\
\hline 8 & $1 / 1$ & - & $\mathrm{M}$ & $>25$ & Desconocida \\
\hline & & & $\mathrm{M}$ & $35-50$ & \\
\hline 9 & $3 / 3$ & Cráneos & $\mathrm{M}$ & $20-35$ & Desconocida \\
\hline & & & $\mathrm{F}$ & $35-50$ & \\
\hline & & & $\mathrm{M}$ & $35-50$ & \\
\hline & & & $\mathrm{M}$ & $35-50$ & \\
\hline 10 & $4 / 4$ & Cráneos & I & $35-50$ & Desconocida \\
\hline & & & I & $35-50$ & \\
\hline & & & $\mathrm{M}$ & $20-35$ & \\
\hline & & & $M$ & $20-35$ & \\
\hline 11 & $5 / 5$ & cráneos y mandíbulas & $\mathrm{M}$ & $35-50$ & Desconocida \\
\hline & & & $\mathrm{M}$ & $35-50$ & \\
\hline & & & $\mathrm{I}$ & $35-50$ & \\
\hline 12 & $1 / 1$ & - & $\mathrm{M}$ & $25-30$ & Desconocida \\
\hline 13 & $1 / 1$ & - & $\mathrm{M}$ & $28-36$ & Prox. de la Misión Salesiana \\
\hline 14 & $1 / 1$ & - & $\mathrm{M}$ & $36-48$ & Ea. San Julio \\
\hline
\end{tabular}


de un individuo (de 4 a 7 individuos), mientras sólo 4 cajas $(28,6 \%)$ incluyen restos que corresponden sólo a un individuo (Tabla 2). Asimismo, las determinaciones de sexo y edad mostraron que 9 cajas (64,3\%) contienen elementos óseos de adultos, mientras las demás incluyen tanto restos de adultos como de subadultos. La composición sexo-etaria de cada una de las cajas, así como el número probable de individuos en cada una de ellas, se presenta en la Tabla 2. Sobre este último punto, las estimaciones de MLNI y MNI arrojaron resultados idénticos para todos los casos, debido probablemente al número reducido de individuos que componen cada caja. Asumiendo que restos de un mismo individuo no forman parte de más de una caja, la colección estaría compuesta por un número mínimo de 51 individuos, de los cuales 43 son adultos y 8 subadultos. A su vez, de los 43 adultos 24 son masculinos, 8 femeninos $y$ 11 indeterminados (Tabla 3). Sin embargo, aunque parece poco probable, la mezcla de individuos entre cajas no puede ser completamente descartada.

Respecto de los estudios paleopatológicos, la colección presenta una amplia variedad de lesiones de diferentes etiologías, algunas de ellas de interés casuístico para la región, de las que se mencionan las más destacadas. Los resultados se presentan en primer lugar para aquellos restos que pudieron ser identificados como correspondientes a un único individuo, y por otra parte para el conjunto de la colección.

Los restos presentes en la Caja Nro. 8 corresponden a un individuo adulto de procedencia desconocida. Es difícil determinar el sexo por ausencia del cráneo y la pelvis, aunque por la robustez de los elementos presentes, particularmente huesos largos, probablemente se trata de un individuo masculino. A partir de la fusión de huesos largos puede considerarse que se trata de un individuo mayor de 25 años al momento de la muerte. En este caso se reconocieron principalmente lesiones osteoarticulares. Entre ellas se identificaron moderadas labiaciones marginales del extremo distal de ambos fémures, particularmente el derecho; labiaciones de ambas rótulas predominantemente la derecha, la cual presenta una marcada porosidad en su carilla articular; y labiaciones del cóndilo externo proximal de la tibia derecha. Estas lesiones evidencian la enfermedad degenerativa articular de ambas rodillas, la derecha de mayor evolución. Se observó un ligero pulido en la superficie articular del fémur y la rótula derecha, aunque bajo lupa no se identificaron las estrías propias de la eburnación y que constituyen un criterio diagnóstico para esta característica. Fue registrada también una osteofitosis de moderado desarrollo en el margen superior anterior de la tercera vértebra lumbar $\left(\mathrm{L}_{3}\right)$, simétrico en todo el cuerpo vertebral. Además, se registró la perforación de la lámina ósea que separa las fosas del olecranon y el coronoides en el extremo distal del húmero derecho (Figura 2). Esta perforación, conocida como apertura septal, ha sido asociada a procesos etiológicos de stress mecánico, aunque también ha sido considerado como un rasgo discontinuo (Capasso et al. 1999). Una investigación reciente propone la primera hipótesis como la más probable, asociada a la acción mecánica del olecranon y el coronoides así como las estructuras musculares y articulares sobre la membrana de la fosa olecraneana (Mays 2008). Finalmente, en los restos de este individuo no fueron observados indicios de stress sistémico (hiperostosis porótica, cribra orbitalia o hipoplasia del esmalte dental), así como tampoco se registraron lesiones de origen infeccioso específico o inespecífico. Las piezas dentales se encuentran con un desgaste moderado, sin caries o abscesos.

Los restos ubicados en la Caja Nro. 12 corresponden a un individuo adulto joven, probablemente masculino, de entre 25 y 30 años de edad al momento de la muerte. También en este caso se desconoce la procedencia de los restos. En los elementos presentes fueron identificadas lesiones redondeadas de $20 \mathrm{~mm}$. de diámetro en las fosas intercondileas de ambos fémures y lesiones del margen supero-posterior de las facetas distales de los

Tabla 3. Composición Total de la Colección.

\begin{tabular}{|c|c|c|c|}
\hline \multicolumn{4}{|c|}{ Número de Individuos por Sexo y Edad } \\
\hline & Masculinos & Femeninos & Indeterminados \\
\hline Adultos & 24 & 8 & 11 \\
\hline Subadultos & - & - & 8 \\
\hline MLNI total & \multicolumn{3}{|c|}{51 Individuos } \\
\hline
\end{tabular}




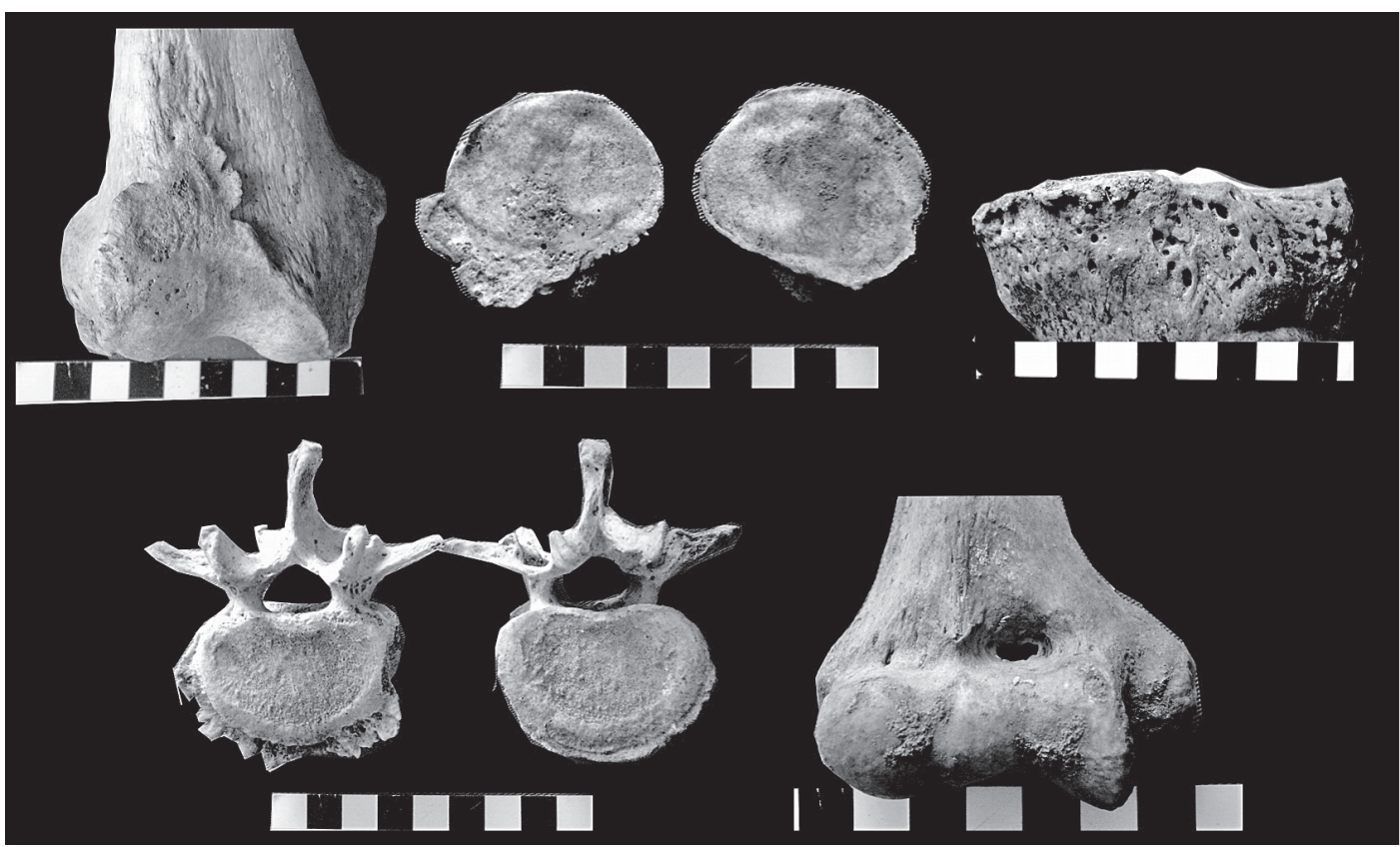

Fig. 2. Lesiones osteoarticulares observadas en restos humanos depositados en la Caja Nro. 8. Arriba: fémur derecho, patelas y tibia derecha, donde se observan labiaciones marginales articulares de moderada evolución particularmente en la rodilla derecha. Abajo izquierda: osteofitos marginales en vertebras lumbares. Abajo derecha: perforación de la fosa del olecranon del húmero derecho.

cóndilos femorales. Estas lesiones son comúnmente reconocidas como el resultado del stress mecánico repetitivo de los músculos gastrocnemios, hiperemia (aumento de la velocidad sanguínea) y la reabsorción ósea localizada (Mann y Hunt 2005), y que han sido asociadas en general a la hiperflexión de la cadera y rodillas (Trinkaus 1975) adoptada durante posturas de acuclillamiento prolongado (Capasso et al. 1999; Dlamini y Morris 2005). Además fueron observadas osteofitosis marginales de moderada evolución en las vértebras de la columna lumbar y primera vértebra sacra, con macroporosidad de las superficies superiores e inferiores de los cuerpos vertebrales (Figura 3). Tampoco en este caso fueron registradas lesiones atribuibles a procesos nutricionales-metabólicos o de origen infeccioso.

Los restos depositados en la Caja Nro. 13 corresponden a un individuo masculino adulto de entre 28 y 36 años de edad. Aunque no fue posible precisar el lugar exacto del hallazgo de estos restos, se obtuvo información que sugiere que fueron recuperados en proximidades de la Misión Salesiana, en cuya excavación participó el propio Padre Tico (Ticó com. per.). En este caso sólo fueron registradas

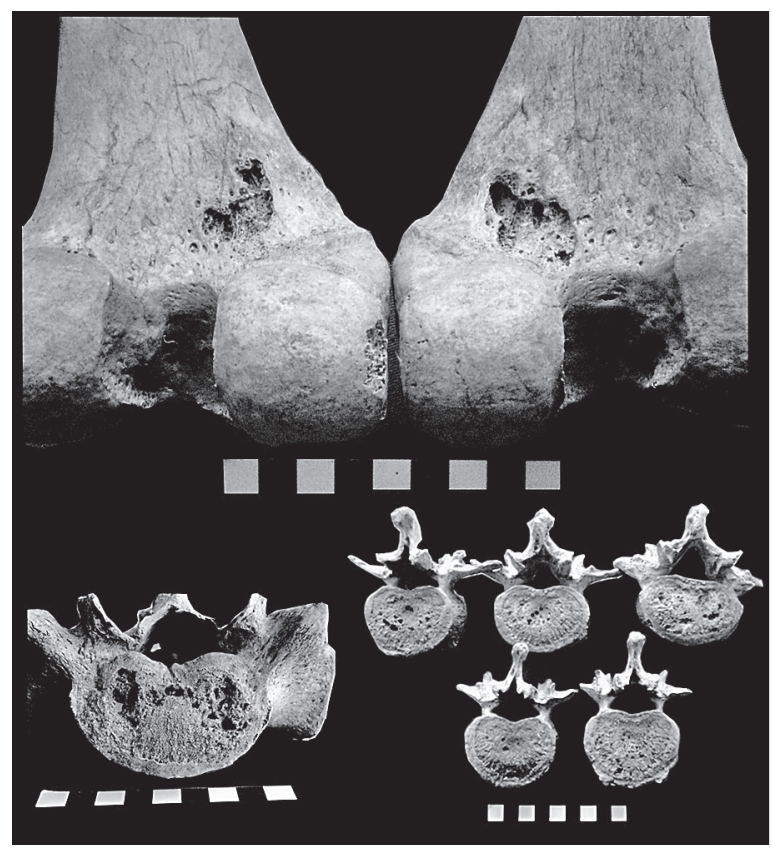

Fig. 3. Lesiones óseas identificadas en el individuo depositado en la Caja Nro. 12. Arriba: lesiones en la fosa intercondilia de ambos fémures, compatibles con lesiones por acuclillamiento. Abajo: macroporosidad de los cuerpos vertebrales lumbares y de la primera vértebra sacra. 


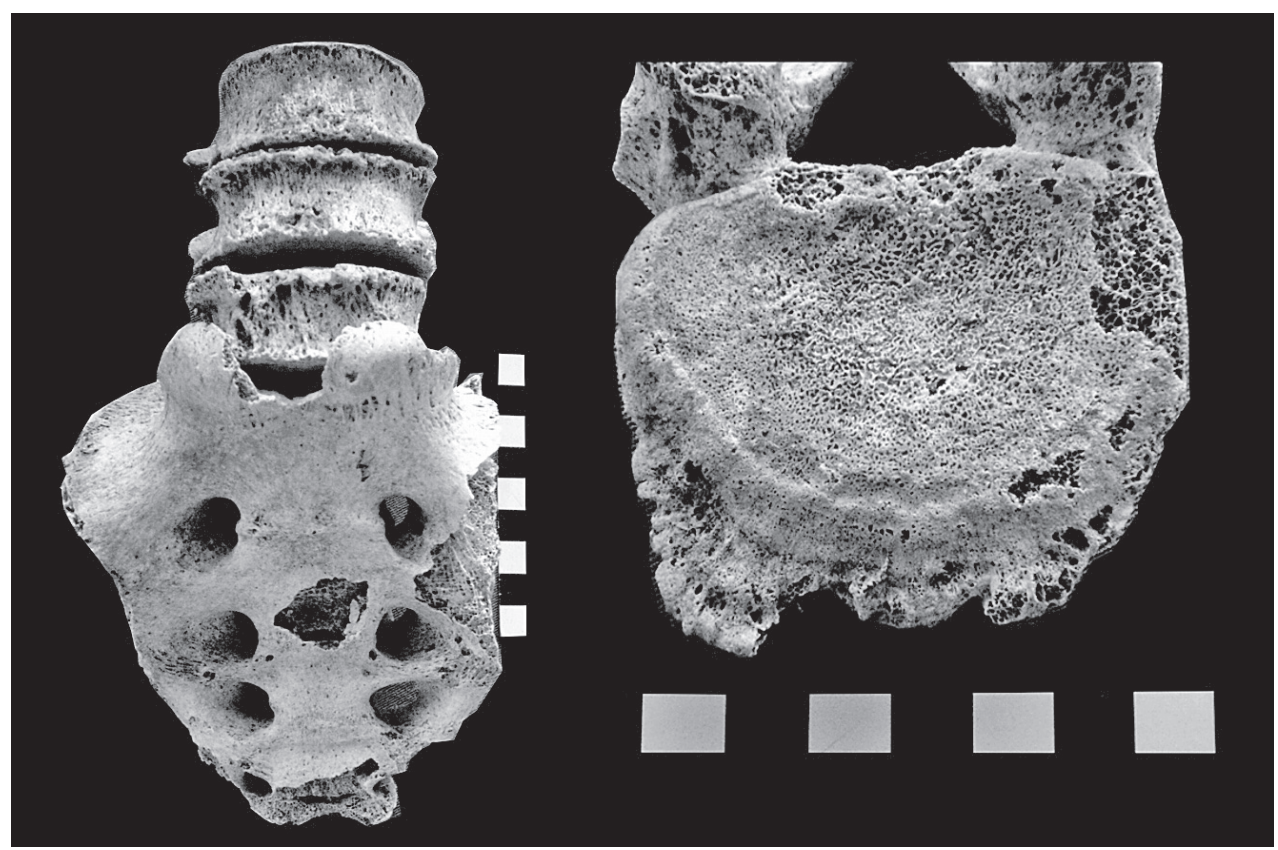

Fig. 4. Osteofitosis de avanzada evolución en vértebras lumbares y primera vértebra sacra del individuo depositado en la Caja Nro. 13.

algunas lesiones de origen osteoarticular. Entre ellas la más relevante incluye una osteofitosis de marcada evolución en la primera vértebra sacra $\left(\mathrm{S}_{1}\right)$, acompañada de importantes osteofitos marginales en los cuerpos vertebrales $\mathrm{L}_{3}, \mathrm{~L}_{4}$ y $\mathrm{L}_{5}$ (Figura 4). Además se identificaron labiaciones de pequeño desarrollo en los cóndilos distales del fémur izquierdo. No se observaron lesiones sistémicas, nutricionales o infecciosas.

El último individuo identificado aisladamente se encuentra depositado en la Caja Nro 14. Se trata de un individuo masculino adulto de entre 36 y 48 años de edad al momento de la muerte y que fue recuperado por aficionados en el año 1996 dentro del espacio ocupado por la Estancia San Julio, en el centro norte de la estepa fueguina. Estos materiales presentan un valor particular por tratarse de uno de los escasos hallazgos correspondientes a esta región. El estudio radiocarbónico realizado sobre una costilla realizado en el Arizona Radiocarbon AMS Facility informó que los restos poseen una antigüedad de 350 \pm 50 años AP (UGAMS3597). A su vez, análisis isotópicos realizados en el Center for Applied Isotope, University of Georgia (UGA) informaron un valor de $\delta^{13} \mathrm{Ccol}$ de $-18,2 ; \delta^{13}$ Cap de $-16,8$ y $\delta^{15} \mathrm{~N}$ de 14,4 (UGAMS3597). Aunque el valor de la relación $\mathrm{C} / \mathrm{N}$ obtenido $(2,85)$ se encuentra algo por debajo de los valores aceptados para muestras sin alteración diagenética (2,9 a 3,6 sensu DeNiro 1985), los valores isotópicos indicarían un consumo predominantemente de recursos terrestres con una ingesta baja de alimentos de origen marino.

El esqueleto se encuentra casi completo, con una conservación de buena a regular debido a la pérdida postmortem de tejido óseo en las epífisis de huesos largos, costillas y pelvis. En este caso se identificaron particularmente lesiones degenerativas articulares. Entre ellas, una entesopatía leve de la epífisis distal de la fíbula derecha, probablemente producida por stress mecánico. Además se registraron osteofitosis de los cuerpos vertebrales $T_{11}$ y $\mathrm{T}_{12}$ con reducción de la altura del cuerpo de la primera de ellas y alteraciones de la morfología de las carillas articulares inferiores y superiores. Se registraron labiaciones marginales moderadas de los cóndilos distales de ambos fémures y porosidad de ambos acetábulos de las pelvis. También se registró una porosidad moderada de la articulación temporomandibular izquierda compatible con un proceso osteoartrósico, acompañada de un moderado desgaste atricional y la pérdida premortem del tercer molar superior izquierdo y el primer premolar 


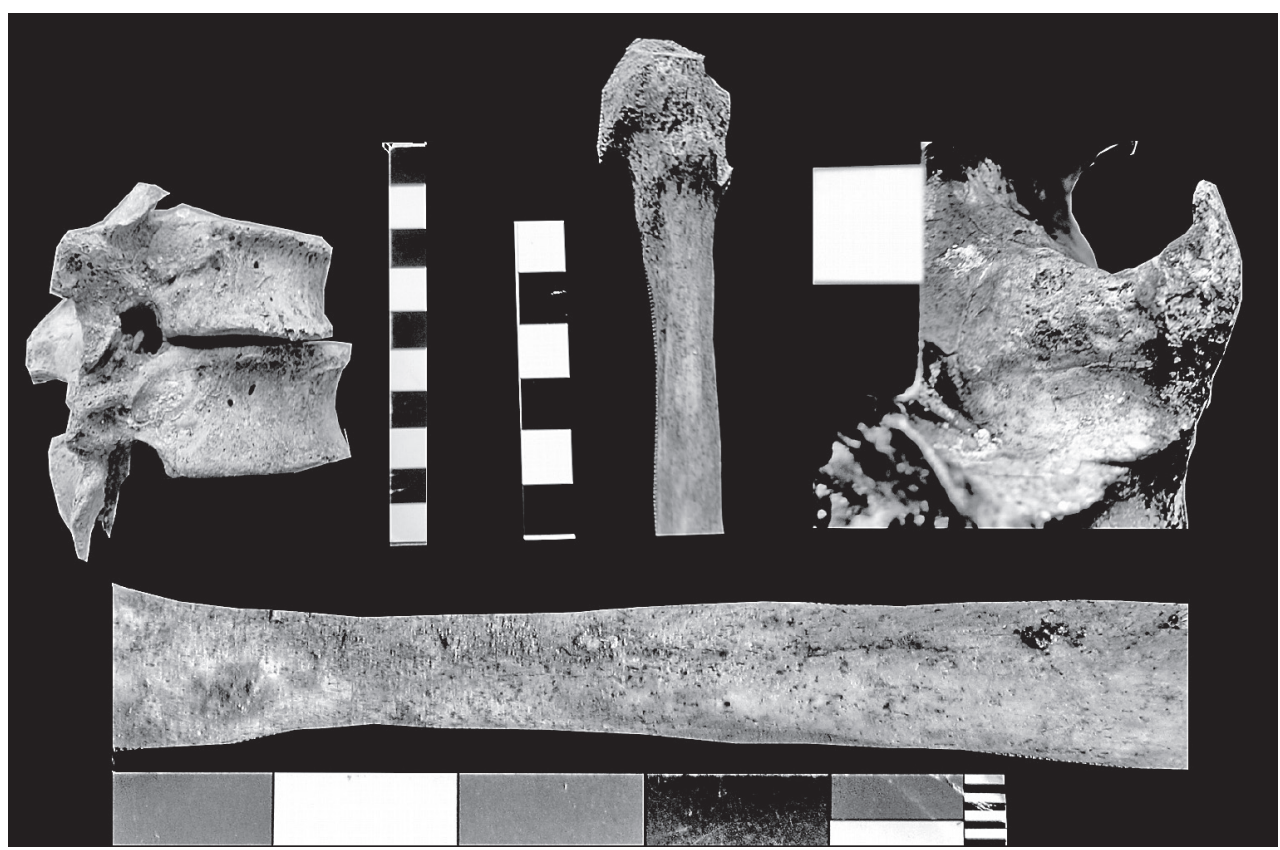

Fig. 5. Lesiones observadas en el individuo depositado en la caja Nro. 14. Arriba izquierda: labiaciones marginales de los cuerpos vertebrales $\mathrm{T}_{11}$ y $\mathrm{T}_{12}$. Arriba centro: entesopatía del extremo distal de la fíbula derecha. Arriba derecha: lesión osteoarticular de la articulación temporomandibular izquierda. Abajo: periostitis activa de la tibia izquierda.

inferior derecho. Finalmente, se observaron reacciones periósticas (periostitis) activas de moderada evolución en ambas fíbulas y tibias (Figura 5) que por sus características y distribución parecen compatibles con procesos infecciosos inespecíficos. No se identificaron lesiones que puedan ser atribuidas a stress sistémico nutricional o metabólico, así como tampoco lesiones de origen traumático.

En el conjunto de la colección fueron identificados dos casos de lesiones traumáticas producidas por proyectiles. Entre los restos ubicados en la caja Nro. 5 se encuentran dos pelvis correspondientes a un mismo individuo masculino en las cuales se identificaron fragmentos de puntas de proyectil, uno en la hemipelvis izquierda y otro en la derecha. El primero atraviesa la cresta iliaca ingresando desde la porción posterolateral izquierda. El otro fragmento se encuentra parcialmente inserto en la porción anterior de la cresta iliaca de la pelvis derecha, con un ángulo de ingreso en sentido anterolateral derecho. La ubicación y dirección de este último proyectil habría implicado lesiones y compromisos de órganos abdominales antes de alojarse en la pelvis. Esta hipótesis, además de la ausencia de remodelación ósea, resulta compatible con el posible

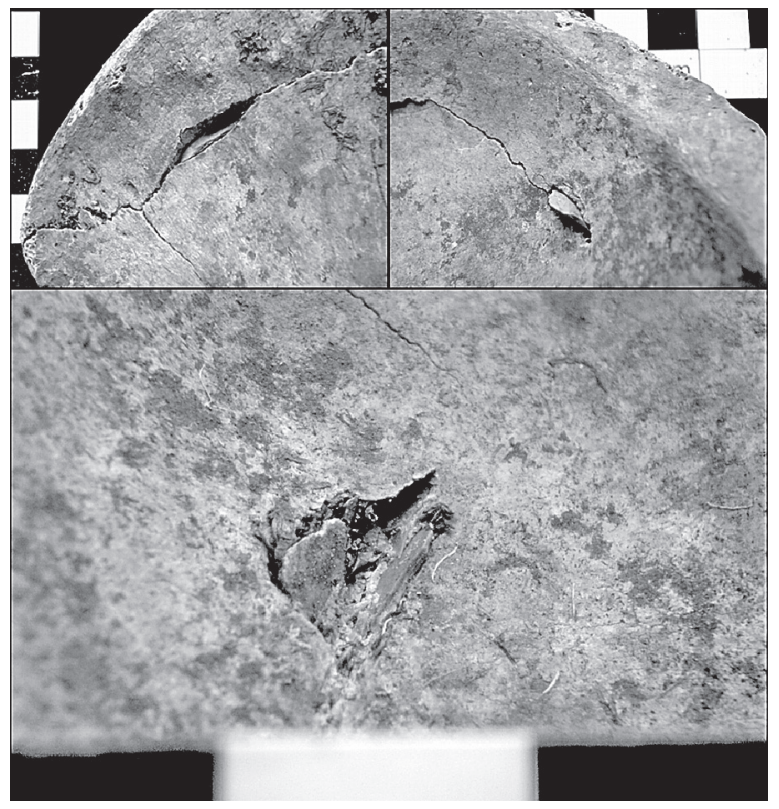

Fig. 6. Lesiones producidas por puntas de proyectiles en ambas pelvis depositadas en la caja Nro. 5. Arriba izquierda: vista posterior de la cresta iliaca izquierda, donde se observa el ingreso del proyectil. Arriba derecha: vista anterior de la creta iliaca donde se observa la punta del proyectil, que atraviesa completamente la estructura ósea. Abajo: vista anterior de la cresta iliaca derecha, donde se observa el ingreso del proyectil.

Nótese en ambos casos la ausencia de remodelación ósea. 


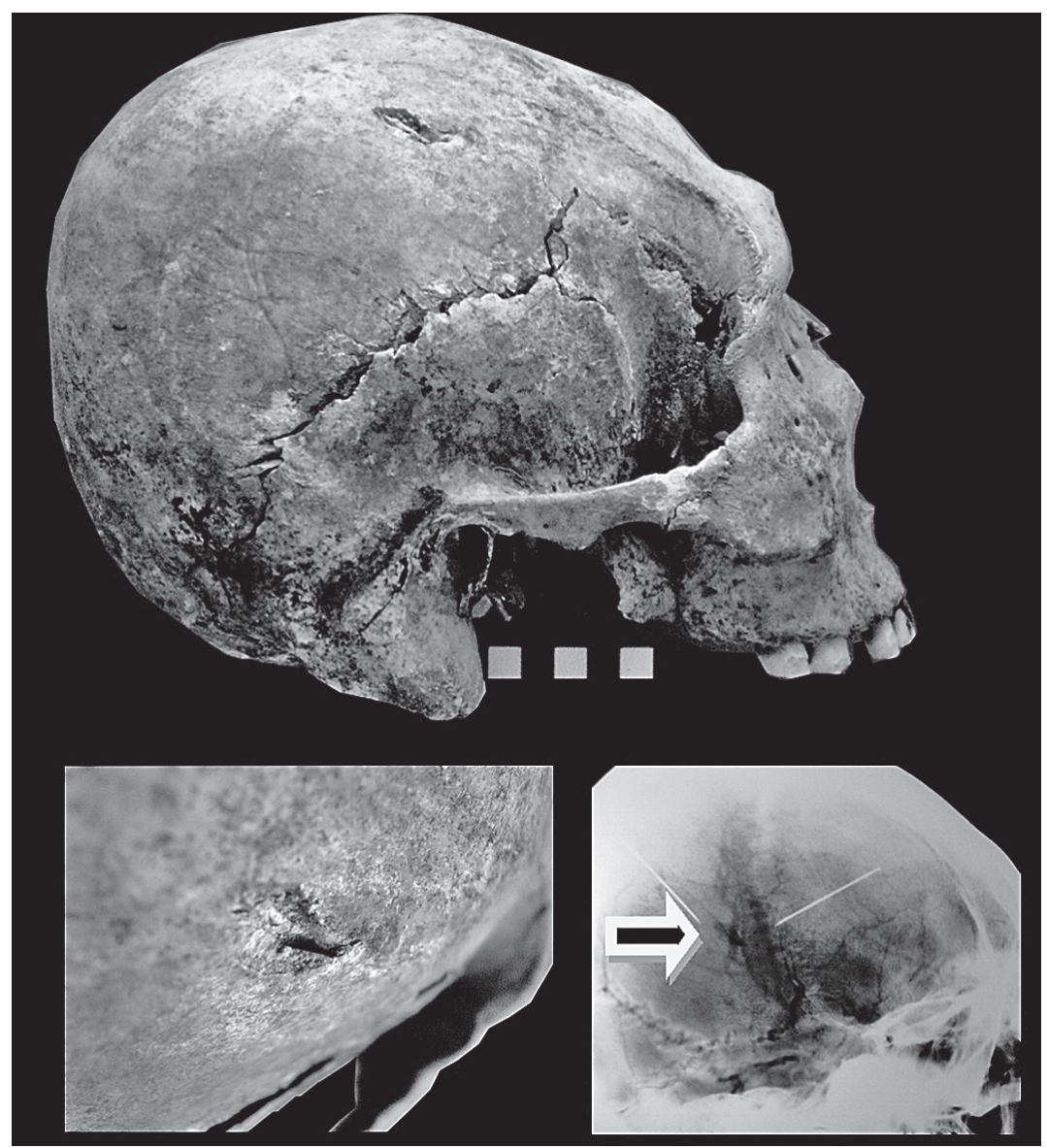

Fig. 7. Lesión traumática perimortem con fragmento de proyectil sobre el parietal derecho de uno de los cráneos depositado en la caja Nro. 9.

origen perimortem de las lesiones, posiblemente vinculadas con un episodio relacionado con la muerte del individuo (Figura 6).

Además, se registró una lesión sobre el parietal derecho de uno de los cráneos ubicados en la caja Nro. 9, correspondiente a un individuo adulto probablemente masculino. La fractura lineal de $15 \mathrm{~mm}$. de longitud en sentido anteroposterior y $7 \mathrm{~mm}$. de longitud en sentido mediolateral, presenta la inclusión de un pequeño fragmento de proyectil lítico en trayecto oblicuo a la tabla craneana. Una imagen radiográfica lateral tomada sobre el cráneo permitió observar la lesión, aunque no aportó mayor información sobre el proyectil (Figura 7). No se evidenció remodelación en las márgenes de la lesión, por lo que podría tratarse de una lesión perimortem. Sin embargo, los estudios radiográficos no mostraron perforación de la tabla craneana y no se dispone de otros elementos óseos pertenecientes a este individuo que pudieran apoyar esta hipótesis.

Entre los marcadores inespecíficos de stress sistémico, sobre un total de 21 cráneos presentes se registraron 12 cráneos (57\%) con indicios de hiperostosis porótica o cribra orbitalia. Entre ellos, un caso notable es el cráneo presente en la Caja Nro. 2 que mostró un desarrollo activo avanzado de cribra orbitalia (Figura 8). A pesar de la marcada cribra observada, no se registró hiperostosis en la calota. Aunque la relación entre lesiones ubicaciones en la calota y en el techo de las orbitas no está aún aclarada, las primeras parecen estar principalmente vinculadas con procesos crónicos a largo plazo (Stuart-Macadam 1989, 1998). De ser así, la presencia de este marcado hallazgo sólo sobre el techo de las orbitas podría implicar el desarrollo agudo de la anemia. 


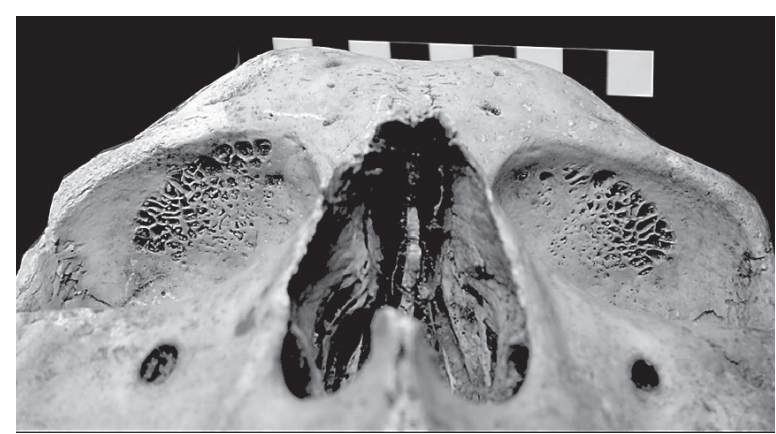

Fig. 8. Cribra orbitalia de gran evolución identificada en el cráneo depositado en la caja Nro. 2.

Sólo ocho elementos óseos presentaron reacciones periósticas. Como se mencionó, cuatro de ellos corresponden a elementos de miembros inferiores del individuo ubicado en la Caja Nro. 14 , probablemente como resultado de procesos infecciosos inespecíficos. Los restantes casos corresponden a tres lesiones halladas en el tercio medio de tibias depositadas en la Caja Nro. 7 y una lesión registrada en una tibia ubicada en la Caja Nro 1. En ambos casos no fue posible descartar durante el diagnóstico diferencial si las lesiones son el resultado de procesos infecciosos o traumatismos menores localizados sobre estos elementos.

\section{CONCLUSIÓN Y COMENTARIOS FINALES}

Los trabajos realizados sobre la muestra osteológica depositada en el museo de la antigua Misión Salesiana "La Candelaria" permiten por un lado, continuar con las tareas de puesta en valor y mejora de las condiciones de conservación de los restos óseos humanos que la componen. En este sentido, se complementan con estudios previos desarrollados sobre otras colecciones de Patagonia Austral (Guichón y Suby 2006; Guichón et al. 2006; Suby et al. 2009). Por otro lado, aporta una serie de información contextual y de conformación de la muestra compuesta por un número mínimo de individuos relevante que resultará de utilidad para futuros trabajos bioarqueológicos y paleopatológicos de mayor extensión. Finalmente, incluye algunos casos patológicos de interés para la región debido a que no han sido reportados anteriormente o representan patologías poco frecuentes.

Desde un punto de vista paleopatológico, en este trabajo no se realizaron estudios de preva- lencia de lesiones patológicas y su relación con la composición sexo-etaria de la muestra, dado que se trata de un conglomerado de datos en su mayoría sin información de procedencia, contextualización $y$ en el cual los restos esqueletales se encuentran en general no individualizados. En este sentido, optamos por un análisis descriptivo que permita generar expectativas y tendencias, dado que un análisis cuantitativo de las lesiones podría generar sesgos importantes que dificulten la interpretación final. En este contexto, los estudios permitieron reconocer que las lesiones más abundantes en la muestra fueron las asociadas a stress mecánico y enfermedades degenerativas articulares de leve a moderada evolución que afectaron particularmente a elementos de la columna vertebral, articulaciones de huesos largos de miembros inferiores y articulaciones de la pelvis. La mayor frecuencia de este tipo de lesiones suele ser recurrente en poblaciones cazadoras recolectoras (Ortner 2003). Aunque no se dispone de trabajos específicos para las poblaciones patagónicas, los estudios realizados hasta el momento presentan en general una predominancia de lesiones osteoarticulares (Guichón 1994; Constantinescu 1999; Garcia Guráieb 2004, 2006; Suby 2007). De igual manera un elevado porcentaje (57\%) de los cráneos que componen la muestra presentaron evidencias de hiperostosis porótica o cribra orbitalia, resultado que coincide con la alta frecuencia observada previamente en Patagonia por Guichón (1994). Asimismo, resultaron poco representadas en general las lesiones atribuibles a procesos infecciosos inespecíficos. Finalmente, no se detectaron lesiones patológicas sobre restos correspondientes a subadultos. En general este hecho ha sido explicado como resultado de la escasa respuesta inmunológica de este grupo etario ante patologías de origen infeccioso, lo que podría implicar la evolución aguda de las enfermedades y por lo tanto un menor registro de las lesiones sobre los restos esqueletales (Ortner 1998, 2003).

Probablemente las lesiones más relevantes observadas en la colección corresponden a aquellas de origen traumático con inclusiones de proyectiles. En los dos casos presentados la interpretación más probable se orienta a posibles episodios de violencia interpersonal, un tipo de lesiones que ha sido reportado sólo en algunos casos en Tierra del Fuego (Suby et al. 2008) y en el sur de Santa Cruz 
(Constantinescu 2003). Por el contrario, en el noreste de Patagonia las lesiones traumáticas craneofaciales por armas han sido halladas con mayor frecuencia y están siendo estudiadas sistemáticamente. Barrientos y Gordon (2004) y Gordon (2009) han propuesto que probables incrementos locales en la densidad demográfica durante momentos tardíos podrían haber creado las condiciones para el aumento en los niveles de violencia interpersonal. Si se considera como posible un proceso similar en el sur de Patagonia, es posible plantear como hipótesis que los restos estudiados aquí podrían corresponder a momentos tardíos del poblamiento. Sin embargo, hasta el momento no disponemos de evidencias suficientes que permitan fortalecer o descartar esta hipótesis.

Considerando los aspectos conservacionistas planteados por Guichón et al. (2008), las situaciones de riesgo a las cuales está asociado el registro bioarqueológico incluyen no sólo los procesos que actúan en momentos previos a su recuperación arqueológica sino también las condiciones de conservación de las colecciones. En relación con estas últimas, la falta de un manejo adecuado ya sea por escasez de recursos o conocimiento, constituye en muchas ocasiones fuentes de descontextualizaciones y destrucción de parte del registro. Aún cuando en Patagonia el estudio de los restos óseos humanos que conforman las colecciones de los museos han sido una fuente importante de información para el estudio de los estilos de vida y la salud de las sociedades del pasado (Aspillaga et al. 1999; Constantinescu 1999; Schinder y Guichón 2003; Aspillaga et al. 2006; Guichón y Suby 2006), las tareas de conservación de colecciones representan un aspecto no siempre tenido en cuenta por los proyectos de investigación y que condiciona la potencial información disponible. Esta fase de las investigaciones implica intervenciones, que aún siendo de bajo costo, pueden ayudar a mantener la integridad y minimizar los daños sobre las colecciones y en las que necesariamente se incluye la participación de administradores, investigadores, comunidades de pueblos originarios y docentes. Aunque las resoluciones para los problemas de conservación que actualmente enfrentan muchas de las colecciones de restos óseos humanos en Patagonia no son simples, es claro que estas tareas deberán continuar en el futuro para garantizar el mantenimiento de este patrimonio no renovable a lo largo del tiempo.

\section{AGRADECIMIENTOS}

Agradecemos a las autoridades de la Escuela Agrotécnica Salesiana "Nuestra Señora de La Candelaria", especialmente al Padre Tico, quien facilitó el acceso a la colección estudiada en este trabajo. A la Dra. Patricia Padinger y personal técnico del Hospital Río Grande, quienes posibilitaron la realización de imágenes radiográficas. A dos revisores anónimos que ayudaron considerablemente a mejorar el manuscrito inicial. Agradecemos muy especialmente a la Lic. Mariana Segura (Equipo Argentino de Antropología Forense), por sus valiosos comentarios en las etapas finales del manuscrito acerca de las determinaciones de sexo, edad y número de individuos. Este trabajo fue financiado por el Proyecto "Paleoepidemiología del Contacto Aborigen Europeo en Patagonia Austral" (PICT 01520).

\section{BIBLIOGRAFÍA}

ADAMS BJ y KONIGSBERG LW. 2004. Estimation of the Most Likely Number of Individuals from Commingled Human Skeletal Remains. American Journal of Physical Anthropology 125:138-151.

ASPILLAGA E, OCAMPO C, RIVAS P. 1999. Restos Óseos Humanos de Contextos Arqueológicos del Área de Navarino: Indicadores De Estilo De Vida En Indígenas Canoeros. Anales Del Instituto De La Patagonia 26:123-136.

ASPILLAGA EF., CASTRO MD. , RODRIGUEZ M y OCAMPO CE. 2006. Paleopatología y estilo de vida: el ejemplo de los Chonos. Magallania 34(1):77-85.

BARRIENTOS G y GORDON F. 2004. Explorando la relación entre nucleamiento poblacional y violencia interpersonal durante el Holoceno tardío en el noreste de Patagonia (República Argentina). Magallania 32:53-69.

BASS WM. 1995. Human Osteology. A laboratory and field manual. Special Publication No. 2 of the Missouri Archaeological Society. Columbia. Third Edition, Fifth printing.

BUIKSTRA JE. y UBELAKER DH. 1994. Standars for data collection from human skeletal remains. Arkansas Archaeological Survey Research Series N44. Arkansas, USA.

CAMPILLO D. 2001. Introducción a la Paleopatología. Bellaterra Arqueología. España.

CAPASSO L, KENNEDY KAR y WILCZAK CA. 1999. Atlas of Occupational Markers on Human Remains. Journal of 
Paleontology. Monographic Publicacion 3. Edigrafital S.p.A. Teramo. Italia.

COCILOVO JA y GUICHÓN RA. 1985/1986. Propuesta para el estudio de las poblaciones aborígenes del extremo austral de Patagonia. Anal. Inst. Pat., Univ. Magallanes, Serie Cien. Soc. 16:111-123, Punta Arenas.

CONSTANTINESCU F. 1999. Evidencias bioantropológicas para modos de vida cazador recolector terrestre y marítimo en los restos óseos humanos de Tierra del Fuego. Anales del Instituto de la Patagonia (Serie Ciencias Humanas) 27:137-174.

CONSTANTINESCU F. 2003. Obsidiana verde incrustada en un cráneo Aónikenk: ¿tensión social intraétnica...o interétnica? We'll never know! Magallania 31:149-153

DeNIRO MJ. 1985. Postmortem preservation and alteration of in vivo bone collagen isotope ratios in relation to paleodietary reconstruction. Nature 317: 806-809.

DLAMINI N. y MORRIS AG. 2005. An investigation of the frequency of squatting facets in later stone age foragers from South Africa. International Journal of Osteoarchaeology 15:371-376

GARCIA GURAIEB S. 2004. Salud y enfermedad en cazadoresrecolectores del Holoceno tardío en el Lago Salitroso (Santa Cruz). Tesis de Licenciatura en Ciencias Antropológicas (orientación en Arqueología). Facultad de Filosofía y Letras, Universidad Nacional de Buenos Aires. MS.

GARCIA GURAIEB S. 2006. Salud y enfermedad en cazadoresrecolectores del Holoceno tardío en la cuenca del Lago Salitroso (Santa Cruz). Intersecciones en Antropología 7:37-48.

GARCIA MORO C, HERNANDEZ M y LALUEZA C. 1997. Estimation of the Optimun Density of the Selk'nam from Tierra del Fuego: Inferences About Human Dynamics in Extreme Environments. American Journal of Human Biology 9:699-708.

GORDON F. 2009. Atribución causal a traumas craneofaciales en muestras del norte de Patagonia (República Argentina): una perspectiva experimental. Magallania 37(2):57-76.

GRAY H. 1949. Anatomía Humana Descriptiva y Aplicada. Vigésima octava edición inglesa. Johnston TB y Whillis J Eds. Emecé, Buenos Aires.

GUICHÓN RA. 1994. Antropología Física de Tierra del Fuego, Caracterización Biológica de las Poblaciones Prehispánicas. Tesis Doctoral. presentada en la Facultad de Filosofía y Letras de la Universidad de Buenos Aires. MS.

GUICHÓN RA y SUBY JA. 2006. La colección del Museo de Historia Natural de Londres correspondientes a restos óseos humanos de Patagonia Austral. Chile. Magallania 34(2):47-56.
GUICHÓN RA, SUBY JA, CASALI R y FUGASSA MH. 2006.

Health at the time of native-european contact in Southern Patagonia. Memorias do Instituto Oswaldo Cruz 101 (Suppl. II): 97-105.

GUICHÓN RA, SUBY JA Y FUGASSA MH. 2008. El Registro Biológico Humano en Patagonia Austral. Algunas Líneas de Análisis. En Arqueología de la Costa Patagónica. Perspectivas para su Conservación. Editoras: Cruz I., y Caracotche MS. Universidad Nacional de la Patagonia Austral y Secretaría de Cultura de la Provincia de Chubut. pp. 232-248.

ISCAN MY, LOTH SR y WRIGHT RK. 1984. Metamorphosis at the sternal rib end: a new method to estimate age at death in white males. American Journal of Physical Anthropology 65:147-156.

LOVEJOY CO, MEINDL RS, PRYZBECK TR Y MENSFORTH RP. 1985. Chronological metamorphosis of de auricular surface of the ilium: a new method for the determination of age at death. American Journal of Physical Anthropology 68:15-28

MANN RW y HUNT DR. 2005. Photographic Regional Atlas of Bone Disease. Charles C. Thomas Publisher. Springfield (USA).

MAYS S. 2008. Septal aperture of the humerus in a medieval human skeletal population. American Journal of Physical Anthropology 136:432-440 (2008)

ORTNER D. 1998. Male-female inmune reactivity and its implications for interpreting evidence in human skeletal paleopathology. En Sex and Gender in Paleopathological Perspective. Grauer AL y StuartMacadam P Eds. Cambridge University. Cambridge, UK. pp 79-92.

ORTNER DJ. 2003. Identification of Pathological Conditions in Human Skeletal Remains, 2nd ed. Elsevier Science/ Academic Press, New York.

SCHINDER G y GUICHÓN RA. 2003. Isótopos estables y estilo de vida en muestras óseas humanas de Tierra del Fuego. Magallania. Anales del Instituto de la Patagonia, Ciencias Humanas 31:33-44.

STUART-MACADAM PL. 1989. Porotic Hyperostosis: relationship between orbital an vault lesions. American Journal of Physical Anthropology 74(4):511-20.

STUART-MACADAM PL. 1998. Iron deficiency anemia: exploring the difference. En Sex and Gender in Paleopathological Perspective. Grauer AL y Stuart-Macadam P Eds. Cambridge University. Cambridge, UK. pp 45-63.

SUBY JA. 2007. Propiedades estructurales de restos humanos y Paleopatología en Patagonia Austral. Tesis Doctoral. Universidad Nacional de Mar del Plata. MS. 
SUBY JA, SALEMME M y SANTIAGO F. 2008. Análisis paleopatológico de los restos humanos del sitio Puesto Pescador 1 (Tierra del Fuego). Magallania 36(1):53-64 SUBY JA., GUICHON RA. Y AF. ZANGRANDO. 2009. El Registro biológico humano de la costa meridional de Santa Cruz. Primeros resultados. Revista Argentina de Antropología Biológica (en prensa).
TRINKAUS E. 1975. Squatting among the Neandertals: a problem in the behavioral interpretation of skeletal morphology. Journal of Archaeological Science 2:327-351

UBELAKER DH. 1989. Human skeletal remains: Excavation, analysis and interpretation. 2nd ed. Washington DC, Taraxacum Press. 